\title{
Liderazgo y cultura organizacional en instituciones de Educación Básica
}

\author{
Pirela de Faría, Ligia del Carmen *
}

\begin{abstract}
Resumen
El propósito de esta investigación fue determinar la relación entre liderazgo y cultura organizacional en instituciones educativas, basados en los teóricos Burns (2003), Bass y Avolio (1994) y Schein (1992). Se trabajó bajo un enfoque cuantitativo, con una metodología descriptiva correlacional, en la población de directivos y docentes de las escuelas públicas de educación básica del Municipio Maracaibo, de donde se extrajo una muestra de 278 a través de un muestreo polietápico, por conglomerado estratificado. Los resultados indican una autopercepción y percepción del líder media alta para el liderazgo transformador y sus resultados, y media baja para el liderazgo transaccional. La cultura se percibe medio alto en formalidad, dirección, integración organizacional, desarrollo de recursos humanos, sistemas de apoyo, compromiso, orientación a resultados, recompensas y beneficios. Se encontraron las correlaciones bajas y algunas significativas entre la cultura organizacional y el liderazgo desde el punto de vista del líder, mientras que desde el punto de vista del seguidor fueron medias, y significativas. Por lo tanto se concluye que existe una adecuada percepción del liderazgo y la cultura, las cuales están relacionadas.
\end{abstract}

Palabras clave: Liderazgo, Cultura organizacional, educación básica.

\section{Leadership and Organizational Culture in Basic Education Institutions}

\author{
Abstract \\ The purpose of this research was to determine the relationship between leadership and organ- \\ izational culture in educational institutions, based on the theoretical postulates of Burns (2003), Bass \\ and Avolio (1994) and Schein (1992). A quantitative approach was used, with descriptive correla- \\ Recibido: 10-12-08. Aceptado: 25-07-10 \\ * Psicóloga. Especialista en Metodología de la Investigación. Magíster en Gerencia de Empre- \\ sas. Doctora en Ciencias Humanas. Profesora titular del Departamento de Investigación de la \\ Facultad de Humanidades y Educación. Universidad del Zulia. PPI Nivel I. 0261-7596259 / \\ 0414-9679691. E-mail: ligiapirela@hotmail.com - fariapirela@cantv.net
}


tional methodology, on the population of principals and teachers at public basic education schools in the City of Maracaibo, where a sample of 278 was extracted through multistage, stratified cluster sampling. Results indicate a medium high self-perception and perception of the leader for transformative leadership and its results, and medium low perception for transactional leadership, with significant differences between the two perceptions. The culture is perceived as medium high with regard to formality, direction, organizational integration, human resource development, support systems, commitment, orientation toward results, rewards and benefits. Low and some significant correlations were found between organizational culture and leadership from the leader's perspective, while from the follower's perspective, they were average and significant. It is therefore concluded that an adequate perception of leadership and culture exists and that they are related.

Key words: Leadership, organizational culture, basic education.

\section{Introducción}

La educación debe contribuir con la formación de las personas, con el fin de mejorar la calidad de vida, el uso racional de los recursos naturales y la conservación del ambiente; de allí que las instituciones educativas sean centros para el desarrollo de la personalidad donde se afianzan valores, como la honestidad, el respeto a los demás, el trabajo perseverante, la solidaridad, el espíritu crítico y la creatividad.

Para lograr tales propósitos es necesario que estas instituciones se desarrollen continuamente a través de un liderazgo transformador que pueda impulsar un cambio en la cultura organizacional. En consecuencia, se requiere analizar ambos aspectos, los cuales son objeto de esta investigación, con el fin de conocer limitaciones y proponer acciones que permitan transformarlas, de manera de mejorar la calidad de la educación asumiendo la responsabilidad del proceso de aprendizaje, impulsando los cambios necesarios en la sociedad y rescatando los valores de identidad nacional.

La nueva dinámica social se caracteriza por la diversidad, los cambios y la crisis, circunstancia que requiere de un li- derazgo con facultades para propiciar la colaboración con un enfoque social, que propenda al desarrollo de los otros y que comparta el éxito de los logros alcanzados. Así pues, los líderes deben evolucionar con valores como la integridad y la habilidad para el trabajo en equipo, crear una visión futura, diseñar una arquitectura social que dé forma a la cultura; deben ser capaces de inspirar y motivar a sus seguidores, de desarrollar cualidades personales y de propiciar el cambio.

En este sentido, Gordon (1997) concibe la cultura organizacional como un sistema compartido de valores, creencias y hábitos dentro de la organización que interactúan con la estructura formal para producir normas de comportamiento. En consecuencia, es parte importante de la institución e incluye la misión, la visión, los valores, la comunicación, y aún siendo un factor intangible, ejerce gran influencia en todos los aspectos de una organización, desde las relaciones humanas hasta la productividad.

En cuanto al aspecto precedente, las instituciones de educación básica, deben propiciar la participación e identificación de los docentes con la misma, y no se limiten a la labor educativa con una ac- 
Liderazgo y cultura organizacional en instituciones de Educación Básica

Pirela de Faría, Ligia del Carmen

titud pasiva ante los cambios. De igual modo hacer énfasis en el logro de los objetivos y metas planteadas con una cultura proactiva caracterizada por la autoevaluación y autogestión institucional.

Por lo tanto, el liderazgo de los directivos y docentes de estas instituciones obliga a plantearse una revisión mediante la cual se comprueben la validez, la aplicación y la utilidad de las concepciones existentes, para la generación de cambios en la cultura organizacional, que a su vez conduzcan a realizar transformaciones en la sociedad.

Este trabajo se plantea, entonces, determinar la relación entre el liderazgo y la cultura organizacional con el fin de identificar oportunidades de mejora en relación a estas variables, para su desarrollo en las instituciones de educación básica. Para ello, se aplicó un enfoque cuantitativo y una metodología correlacional, descriptiva y de campo (Hernández, Fernández y Baptista, 2000).

La población objeto de este estudio fue el personal que trabaja en las instituciones públicas de educación básica del municipio Maracaibo, conformada por 8.258 personas, entre directivos (Director y subdirector) y personal docente. Se aplicó un muestreo probabilístico polietápico, por conglomerado estratificado, seleccionando aleatoriamente las instituciones y luego las personas de acuerdo al cargo que ocupan. Asimismo se aplicó la fórmula de Sierra (1999) para determinar el tamaño de la muestra para poblaciones infinitas, con resultado de 278 personas, con un $97.5 \%$ de confianza y $6 \%$ de error.

Para medir el liderazgo se utilizó el cuestionario Multifactorial de Liderazgo de Bass y Avolio (2004) el cual consiste en dos partes de 45 ítemes, una forma para autoevaluarse como líder y la segunda para evaluar a otra persona como líder como el supervisor o par, en los estilos de liderazgo transformador y transaccional y pasivo - evitación.

Para este estudio se determinó su confiabilidad aplicándolo a una muestra de 50 docentes y directores, resultando 0.94 por el método alpha de Cronbach y de 0.81 por división por mitades para la primera parte (Lider Form), y de 0.94 y de 0.75 para la segunda parte (Seguidor form), respectivamente, por lo que el instrumento tiene alta confiabilidad para ser aplicado a esta población.

Por otra parte para medir la variable Cultura organizacional se diseñó un cuestionario tipo escala con 46 preguntas, con las siguientes alternativas de respuesta: Totalmente en desacuerdo (1), Mediante en desacuerdo (2), Medianamente de acuerdo (3) y Totalmente de acuerdo (4).

Se determinó la validez de contenido a través del juicio de 7 expertos, luego se aplicó a una muestra de 50 docentes seleccionados al azar con el fin de aplicar el análisis discriminatorio de ítems a través de la prueba t de Student, del cual se eliminó la pregunta 7. Posteriormente se determinó la confiabilidad a través del método de división por mitades con resultado de 0.96 y la consistencia interna con la formula Alpha de Cronbach siendo de 0.95 , lo cual indica que el cuestionario tiene una alta confiabilidad. Adicionalmente se le aplicó el análisis factorial con el fin de determinar la validez de constructo del instrumento, encontrándose 11 componentes principales que explican el 85,3\% de la varianza, es decir que los ítems co- 
rrelacionaron significativamente con los indicadores que miden, lo cual demuestra una alta validez.

\section{Aproximaciones teóricas sobre el liderazgo}

Según Lussier y Achua (2002), influir es el proceso en el que el líder comunica sus ideas, los seguidores las aceptan y se sienten motivados para respaldarlas y efectuar el cambio. La influencia es la parte medular del liderazgo, y tiene que ver con la relación que se establece entre líderes y seguidores, donde los primeros se ganan la voluntad y el entusiasmo de los segundos, quienes están dispuestos a que influyan sobre ellos.

Por lo tanto, el liderazgo se entiende como una relación de influencia que ocurre entre los líderes y sus seguidores, mediante la cual las dos partes pretenden llegar a cambios y resultados reales que reflejen los propósitos que comparten.

En este sentido, Bennis y Nanus (1985) plantean que para que el líder ejerza influencia deben estar presentes tres conceptos principales: compromiso, complejidad y credibilidad. Los líderes deben saber comunicar una visión, significado y empuje. Es decir que el liderazgo eficaz mueve organizaciones de estados actuales a futuros, crea visiones de oportunidades potenciales para las organizaciones, inculca a los empleados el compromiso para el cambio y en las organizaciones, nuevas culturas y estrategias que movilizan y concentra la energía y los recursos. Los líderes emergen cuando las organizaciones enfrentan problemas nuevos y complejidades que no pueden resolverse mediante una evolución no guiada. Asumen responsabilidades para reformar las prácticas organizacionales, adaptándolas a los cambios del ambiente. Superan la resistencia al cambio creando visiones de futuro que evocan la confianza y el dominio de nuevas prácticas organizacionales.

En consecuencia, en el presente trabajo se entiende el liderazgo como el proceso mediante el cual un individuo influye sobre la conducta de los demás con el fin de lograr un objetivo, y para ello debe tener unos valores y características, así como realizar una serie de tareas como las que se mencionan a continuación: tener una visión que inspire, aplicar los medios para alcanzar esa visión, estructurar las situaciones grupales, manejar las relaciones interpersonales, la comunicación, el modelaje, congruencia entre lo que dice y lo que hace, capacidad para tomar decisiones, resolver problemas, manejar los conflictos, delegar, empoderizar, iniciar y mantener cambios en la organización, realizar tareas desafiantes, perseverar, desarrollar a los seguidores, asumir la responsabilidad, ser proactivos y creativos, entre otros.

Entre los enfoques contemporáneos para el estudio del liderazgo, destacan el carismático y el transformacional o transformador, los cuales según Daft (2006) son dos estilos que están fundamentados en las características personales de cada líder. Los líderes carismáticos tienen un efecto emocional en las personas porque apelan a su mente y su corazón, crean un ambiente de cambio, articulan una visión idealizada del futuro, se comunican con claridad, inspiran fe y esperanza, y corren riesgos personales para influir en los seguidores. El carisma 
Liderazgo y cultura organizacional en instituciones de Educación Básica

Pirela de Faría, Ligia del Carmen

puede ser utilizado para provecho de las organizaciones y la sociedad, pero también puede ser peligroso. Los líderes transformacionales también crean un ambiente de cambio y estimulan a los seguidores, a creer en la visión de una transformación de la organización, a ir más allá de su interés personal en busca del bien común.

Lussier y Achua (2002), sin embargo, consideran sinónimos los términos de liderazgo carismático y liderazgo transformacional. En este sentido, el carisma se define como el proceso de influencia en las actitudes y premisas de los integrantes de las organizaciones para que realicen cambios importantes y se comprometan con los objetivos organizacionales. Se considera que los líderes carismáticos poseen cualidades excepcionales que inspiran y motivan a la gente para que realicen mas de lo que harían en circunstancias normales. Es más probable que los líderes con carisma surjan como guías durante períodos de gran crisis social y desempeñen un papel decisivo en dirigir la atención de la sociedad a los problemas que esta enfrenta y a una visión radical que ofrece una solución a dicha crisis.

Por lo tanto, este liderazgo es resultado de los atributos del líder y no solamente de la situación, entre los que se encuentran una visión clara del futuro, habilidades excepcionales de comunicación, honradez, confianza en si mismo, inteligencia, energía y orientación a la acción. Asimismo la mayoría de los teóricos consideran que el carisma es resultado de la percepción de los seguidores y esta es influida no solo por las características y comportamiento del líder sino también por el contexto.
Pese a las aportaciones de los teóricos del carisma al campo del liderazgo, tiene sus limitaciones, dado que se destaca el papel de un líder individual que toma la iniciativa de crear y articular una visión para los seguidores. Sin embargo, en una organización que enfrenta grandes desafíos o conflictos internos graves, debido a valores, aspiraciones ideales encontradas, es más probable que la transformación provenga de procesos de liderazgo compartidos que de un enfoque estrictamente individual. Casi todas las investigaciones descriptivas sobre líderes efectivos proponen que el carisma, en su forma individualizada, no tiene que lograr por fuerza cambios importantes en una organización y mejorar su desempeño. Por lo general un cambio organizacional positivo es resultado del liderazgo transformacional de individuos que no se perciben como carismáticos, en consecuencia describe más a individuos extraordinarios como emprendedores visionarios que establecen una nueva organizacional.

Por lo tanto, el liderazgo transformador se centra en los logros de los líderes, más que en sus características personales y las reacciones de los seguidores. Mientras las organizaciones sigan enfrentando los desafíos globales, mayor será la necesidad de líderes que trabajen y pongan en marcha con acierto estrategias audaces para transformar o alinear a la organización con el nivel de turbulencia ambiental. De manera que es una forma de liderazgo que sirve para cambiar el status quo, pues articula los problemas en el sistema actual y una visión convincente de los que podría ser una nueva organización para los seguidores (Lussier y Achua, 2002). 
En este orden de ideas, Bass y Avolio (1994) identifican dos clases fundamentales de liderazgo "transaccional" y "transformador". Los líderes transaccionales tienden a orientarse a la acción, mientras que los transformadores se inclinan más a utilizar un nivel de visión mas elevado. Algunas situaciones de liderazgo requieren el desarrollo de habilidades básicamente transaccionales mientras que otras precisan de procesos fundamentalmente transformacionales.

El liderazgo transformador se mueve a lo largo de las dimensiones de visión y acción, en oposición a tarea y relación. La visión está relacionada con la creación de imágenes de objetivos futuros, mientras que la acción se relaciona con la ejecución de comportamientos inmediatos. La idea general estriba en que "la visión sin acción no es mas que solo un sueño, mientras que la acción sin visión carece de sentido y aburre".

Por otra parte, Burns (2003) plantea que la relación de la mayoría de líderes y seguidores son transaccionales, donde los líderes contactan seguidores con miras a intercambiar una cosa por otra (trabajo por votos, subsidios por contribuciones a campañas electorales). Este tipo de transacciones comprenden la mayor parte de las relaciones entre líderes y seguidores, especialmente entre grupos, legislaturas y partidos políticos.

En este sentido, señala el autor antes mencionado plantea las siguientes características del liderazgo transaccional:

- Recompensa contingente: El líder controla el intercambio de recompensas a cambio de un buen desempeño reconociendo los logros, negociando estas recompensas a cambio de esfuerzo. Indica que hay que hacer si se quiere obtener la recompensa. Asegura a los colaboradores que tendrán lo que esperan a cambio de su esfuerzo. Otorga menciones especiales a promociones a cambio de trabajo bien hecho.

- Administración por excepción (activo): el líder controla y busca desviaciones de las reglas y normas tomando acciones correctivas. Interviene únicamente cuando los colaboradores de desvían de las expectativas. No trata de cambiar nada mientras todo vaya según lo previsto. No ofrece ningún tipo de apoyo ante cualquier fracaso al cumplir con los estándares.

- Administración por excepción (pasivo): el líder interviene solo si no se satisfacen las normas, asumiendo un estilo laissez-faire, declina de sus responsabilidades y evita tomar decisiones.

Por lo tanto, el liderazgo transformador es más complejo y potente. El líder en este enfoque, reconoce y explota una necesidad o demanda personal existente de un seguidor potencial, buscando motivos potenciales, satisfaciendo altas necesidades, y envolviendo a la persona completa del seguidor. Los líderes toman responsabilidad por sus compromisos, es decir, cumplen lo que prometen. El resultado del liderazgo es una relación de estimulación mutua que convierte seguidores en líderes y puede convertir líderes en agentes morales. Los líderes y seguidores tienen una relación no sólo de poder sino de necesidades, aspiraciones y valores mutuos. En respuesta a los líderes, los seguidores tienen un adecuado cono- 
Liderazgo y cultura organizacional en instituciones de Educación Básica

Pirela de Faría, Ligia del Carmen

cimiento de los programas alternativos propuestos por el líder y la capacidad para escoger entre ellos (Burns, 2003).

Al comparar el liderazgo transformador y el transaccional, Burns (2003) establece que el primero se basa en valores finales (justicia, igualdad y derechos humanos) transmitidos a través de la educación, mientras que el segundo se apoya en valores modales (honestidad, lealtad y respeto) por medio del entrenamiento en el cual puede usarse la coerción.

En este sentido, se describe el liderazgo transformador como un proceso en donde existen altos niveles de moralidad y motivación. Los líderes transformadores buscan elevar el grado de conciencia de sus seguidores basados en ideales y valores mas altos, tales como libertad, justicia, igualdad, paz y humanitarismo, en contraste con emociones mas bajas como ambición, miedo, envidia y odio. De manera que este tipo de liderazgo puede ser visto como un proceso de influencia a nivel micro entre individuos y como un macro nivel de influencia para la movilización de poder con miras a cambiar sistemas sociales y producir reformas institucionales.

Este emerge y siempre retorna a las motivaciones, necesidades, aspiraciones y valores fundamentales de los seguidores, pudiendo producir cambios sociales que satisfaga sus necesidades auténticas a la vez que mide las motivaciones de otros por las de sí mismo.

Por su parte, Hellrriegel y Slocum (1998) señalan que la influencia de los líderes transformadores se deriva de la aceptación personal de sus seguidores a ciertos valores. Los valores proporcionan pautas para la toma de decisiones y la conducta, por lo que los seguidores que comparten los valores del líder no dependen de sus órdenes, al menos no directa y exclusivamente. Estos valores en el liderazgo transformador, se desarrollan en base a tres comportamientos que son el desarrollo de la visión, el desarrollo de un marco de referencia y la administración de las impresiones. Otras explicaciones describen a los líderes transformadores como servidores o dirigentes, en vez de jefes. Por lo tanto, la tarea de un líder transformador es convertir a los subordinados en líderes autodirigidos.

En cuanto al desarrollo de la visión, el líder transformador está dispuesto a correr riesgos, a retar al status quo e imponer nuevos paradigmas que sirven de marco referencial a otros. En este sentido impulsa estrategias de participación para evaluar constantemente nuevos métodos y soluciones a los problemas y aprovechamiento de oportunidades.

La administración de impresiones, por su parte, exige del líder transformador asumir comportamientos positivos mediante el uso bien de formas exageradas, bien de acciones fuera de lo común o de conductas ejemplarizantes, que exalten las emociones en los seguidores y también tácticas creadas para destacar su atractivo hacia ellos. De manera que los líderes transformadores dirigen mediante el ejemplo. Sus acciones se dirigen a expresar y reforzar los valores y creencias de la organización, que coinciden con los suyos propios. Utilizan la comunicación gestual, comportamental y simbólica para, con estos lenguajes alternos a la verbalidad, reforzar sinérgicamente los valores que subyacen en la visión.

En otras palabras, Bass y Avolio (1994) definen a los líderes transformado- 
res primariamente en términos de los efectos del líder sobre sus seguidores, quienes sienten confianza, admiración, fidelidad, respeto hacia el líder y son motivados a hacer mas de los que se espera de ellos. De manera que el líder puede transformar a sus seguidores haciéndoles más conocedores de la importancia y valores de los resultados de la tarea, induciéndolos a trascender sus propios intereses a los intereses de la organización o del grupo, y activándoles sus necesidades de más alto rango. En el liderazgo transformador se plantean los siguientes aspectos:

- Carisma (influencia idealizada): proporciona visión y sentido de misión, origina orgullo, genera respeto y confianza. Otorga a sus colaboradores un sentido de propósito. Constituye un modelo que los colaboradores aspiran a imitar.

- Inspiración: comunica altas expectativas, utiliza símbolos para enfocar, expresando los propósitos importantes de manera sencilla.

- Estímulo intelectual: promueve y enfatiza la inteligencia, racionalidad y solución cuidadosa de problemas. Las ideas del líder estimulan a sus colaboradores a reconsiderar sus propias ideas. Los viejos problemas se ven así con nuevos ojos.

- Consideración Individualizada: da atención personal, trata a cada empleado de manera individual, capacitando, aconsejando.

Así mismo, Dilts (1998) agrega la gestión por objetivos, que proporciona a los colaboradores representaciones claras de los objetivos deseados, así como de los indicadores de su consecución, estimulándolos a desplegar sus propios recursos y capacidades.

De lo antes planteado se puede inferir la importancia de los líderes transformadores en la instituciones de educación básica, con valores y rasgos positivos, que asuman comportamientos ejemplarizantes, que exalten las emociones en los seguidores, los transformen y los impulsen a asumir el liderazgo, para expresar y reforzar los valores y creencias de la organización, que la eleven a estados de excelencia.

De esta manera, las organizaciones deben implantar rápidos y profundos cambios, ser capaces de aceptarlos, ser flexibles para cumplir con las diferentes prioridades y simplificar la estructura organizacional, lo cual facilitará la toma de decisiones y la capacitación de los empleados, requerida para enfrentar los problemas y oportunidades, aumentar el compromiso con las acciones de mejora, derivadas del aprendizaje continuo; aspectos estos clave para convertirse en una organización inteligente. Es por ello que se amerita una cultura fuerte, flexible y compartida por todos sus integrantes.

\section{Cultura organizacional: algunas consideraciones}

Siguiendo el orden de ideas planteadas en la sección anterior, la cultura organizacional es definida por Schein (1992) como un patrón de supuestos básicos inventados, descubiertos y/o desarrollados por un grupo, en la medida que aprende a manejar y resolver sus problemas de adaptación externos y de integración interna. Necesariamente estos factores han de ser transmitidos a los nue- 
Liderazgo y cultura organizacional en instituciones de Educación Básica Pirela de Faría, Ligia del Carmen

vos miembros del grupo, como la manera más correcta de percibir, pensar y sentir en relación con sus elementos socioculturales y problemas emergentes.

En la misma sintonía, Bower (1995) establece que la cultura es un elemento invisible para las personas que pertenecen a las organizaciones, al punto de llegar a convertirse, algunas veces, en la forma de hacer las cosas. Sin embargo, en la raíz de cada cultura están los valores en los que se basa la organización, los cuales pueden ser permanentes y durar muchas décadas o pueden cambiar rápidamente por efecto del entorno o de quienes lo dirigen.

Asimismo, Stoner (1996) conceptualiza la cultura organizacional como una serie de entendidos importantes como normas, valores, actitudes y creencias compartidos por los miembros de la organización. Forma actitudes en los empleados y determina la manera en que la organización interactúa con su entorno. Igualmente, Robbins (2004) la designa como un sistema de significados común entre los miembros, que distingue una organización de otras, donde los valores compartidos se conservan y comparten.

Para Serna (1997) dicho término plantea la manera cómo las organizaciones hacen las cosas, cómo establecen prioridades y dan importancia a las diferentes tareas empresariales, es decir incluye aspectos bien importantes para la empresa, porque influyen en la manera cómo los gerentes resuelven las estrategias planeadas, incluyen valores, creencias y comportamientos que se consolidan y comparten durante la vida empresarial.

En este mismo orden de ideas, Siliceo, Casares y González (1999) definen la cultura como el conjunto (sistema) de valores, tradiciones, creencias, hábitos, normas, actitudes y conductas que dan identidad, personalidad y destino a una organización para el logro de sus fines económicos y sociales. A esto se agrega el conjunto de elementos interactivos fundamentales, compartidos grupalmente, sedimentados a lo largo de la vida de la organización a la cual identifican, por lo que son transmitidos a los nuevos miembros, y que son eficaces en la resolución de los problemas.

En conclusión, puede señalarse que la cultura describe la parte del ambiente interno de una organización, es decir supuestos, creencias y valores, muchas veces tácitos, que comparten sus miembros, distinguiéndolos de otras organizaciones, pues estos guían su funcionamiento, afectando la forma en que las personas interpretan las circunstancias y sus esquemas para organizar y retener la información, que les permiten enfocar sus actos. Es evidente por lo que la referencia a un modelo de presunciones básicas inventadas, descubiertas o desarrolladas por un grupo dado, al ir aprendiendo a enfrentarse con sus problemas de adaptación externa e integración interna, y es transmitida, aprendida y compartida por todos. Por lo que en esta investigación se asumió la definición de Schein (1992).

De esta manera, la cultura se refleja en los comportamientos de sus integrantes, en la forma de producir, organizarse, tomar decisiones, ejecutarlas, y cómo realizan las comunicaciones. La presencia de esta cultura hace posible la sinergia de los esfuerzos realizados, dinamizar el sentido de propósito, enfatizar los valores y dar sentido de pertenencia a la organización. 
En tal sentido, una cultura sólida está determinada por valores compartidos, una comunicación que permita transmisión de mensajes que la identifiquen y un liderazgo que dirija a la organización hacia un nuevo modelo de desarrollo. Es decir, las culturas fuertes, estratégicamente correctas y adaptables tienen repercusiones positivas en la productividad a largo plazo (Gordon, 1997).

En concordancia con lo anterior, la comprensión de la cultura facilita la integración e impide el desperdicio de talentos en las organizaciones, permite conocer las percepciones de la gente en la organización, en relación con los factores que afectan su motivación. En tanto, establece un canal de comunicación y relaciones, fortalece los niveles de adhesión y compromiso con la organización. Se hace necesario entonces, incorporar instrumentos de diagnóstico, los cuales permitirán la identificación de las diferencias y peculiaridades de las diversas áreas de la organización, como requerimiento para trazar planes de acción que permitan potenciar los factores de satisfacción y minimizar las debilidades.

Por otra parte, los elementos relevantes en la cultura organizacional según Schein (1992) son los siguientes:

- Artefactos: considerados como los productos, servicios, patrones de conductas de los miembros de la organización, hábitos y costumbres.

- Valores Adoptados: Motivos que explican la forma en que se hacen las cosas.

- Supuestos Básicos: Creencias y mitos, filosofía de los líderes, supuestos, misión y visión.

Conjuntamente los factores que caracterizan la cultura, de acuerdo con Granel, Garaway y Malpica (1998) son:
- El Desarrollo Gerencial: se refiere al grado en que la organización provee oportunidades para el avance y desarrollo en puestos de mayor jerarquía. Se refleja en: las oportunidades de promoción interna, búsqueda interna para promoción, información de salarios y beneficios, claridad de interés en el desempeño, éxito del desarrollo de personal, oportunidades de desarrollo, promoción de los más competentes, y relación entre remuneración y resultados.

- Estilo Gerencial: Es el patrón de estímulo y apoyo a la iniciativa, y a la apertura para cuestionar opiniones superiores y concretar ideas como: libertad en la toma de decisiones, incentivo a tomar riesgos e innovar, críticas constructivas y conflictos, comunicación vertical, apoyo de la alta gerencia y claridad en los resultados esperados.

- Compensación: Se refiere al grado en que el sistema es percibido como justo, ligado al rendimiento y competitivo en el mercado laboral: equidad externa beneficios, satisfacción con la remuneración, y equidad interna y externa con la compensación.

- Orientación a resultados: Es el énfasis que se da a las responsabilidades individuales y grupales, a la competencia y el logro de retos: responsabilidad en resultados, trabajo y metas como reto, alta exigencia en rendimiento, individuos competentes, efectividad implantando decisiones, y la adecuación talentos y exigencias del puesto.

- Integración Organizacional: Es el grado en que se proveen condiciones para la comunicación efectiva y cooperación para cumplir objetivos, a través de: cooperación y comunicación dentro y entre las unidades, claridad entre cargos, in- 
Liderazgo y cultura organizacional en instituciones de Educación Básica

Pirela de Faría, Ligia del Carmen

formación y comprensión de metas de otras áreas.

Asimismo, Robbins (2004) plantea siete características básicas que captan la esencia de la cultura de una organización:

1. Innovación y correr riesgos: grado en que se alienta a los empleados para que sean innovadores y corran riesgos.

2. Minuciosidad: grado en que se espera que los empleados muestren exactitud, capacidad de análisis y atención a los detalles.

3. Orientación a resultados: grado en que la gerencia se centra en los resultados más que en las técnicas y procedimientos para conseguirlos.

4. Orientación a las personas: grado en que las decisiones de la gerencia toman en cuenta el efecto de los resultados en los integrantes de la organización.

5. Orientación a los equipos: Grado en que las actividades laborales se organizan en equipos más que individualmente.

6. Agresividad: grado en que las personas son osadas y competitivas, antes que despreocupadas.

7. Estabilidad: grado en que las actividades de la organización mantienen el estado de cosas, en lugar de crecer.

Todas estas características se presentan en un conjunto de bajo a alto, por lo tanto al evaluar la organización en estas siete características se observa un cuadro realista de su cultura.

Por otro lado, Serna (1997) indica que los factores más influyentes en el desarrollo de la cultura organizacional son:

- Los fundadores: que establecen los incentivos, principios, prioridades, comprensión, valores.
- El estilo de Dirección: Fija el tono de las interacciones, influye en la comunicación, toma de decisiones. Puede ser autocrático - democrático - laissez faire. Establece las Normativas, el Control, participación, grado de permisividad, centralización, puede enfatizar en el trabajo individual o grupal, en su integración o desintegración.

- Claridad en los principios Organizacionales: responde al Grado en que se encuentran explícitos.

- Autonomía Individual: Grado de responsabilidad, independencia y creatividad permitida. centralización - descentralización.

- Estrategia: estructura, normas y procedimientos, supervisión y control. Las estructuras planas son más flexibles, hay mayor comunicación, menos reglas y procedimientos.

- Sistema de Apoyo: Infraestructura de apoyo, información tecnología y telecomunicaciones.

- Sistemas de recompensa, reconocimiento y sanciones: dependen de la Evaluación, promoción, remuneración y ascenso.

- Estimulo al riesgo: tienen que ver con innovación y riesgo calculado.

- Direccionamiento estratégico: de acciones a corto plazo y operativas.

- Talento Humano: característica en cuanto a la educación, experiencia, pertenencia, satisfacción, compromiso.

Integrando las características antes presentadas, en la presente investigación se establecieron las siguientes dimensiones para describir la cultura organizacional de las instituciones educativas:

- Formalidad: Claridad de la estructura, objetivos, metas, procedimientos y responsabilidades. 
- Estilo de dirección: Estímulo, confianza, participación en la toma de decisiones y comunicación.

- Integración organizacional: comunicación lateral, cooperación intra e intergrupos.

- Desarrollo del recurso humano: Evaluación, adiestramiento, oportunidades de crecimiento.

- Compensación y recompensas: equidad interna y externa, beneficios, estabilidad laboral, relación de las recompensas con el desempeño.

- Sistemas de apoyo: Condiciones de trabajo, recursos y tecnología para llevar a cabo el trabajo.

- Compromiso: Sentido de pertenencia a la organización, satisfacción con el trabajo.

- Orientación a resultados: preocupación por alcanzar los objetivos y metas.

A continuación se presentan los resultados encontrados luego de aplicar las estadísticas descriptivas para las variables liderazgo y cultura organizacional, utilizando medidas de tendencia central (promedio) y dispersión (desviación, valor mínimo y máximo), y las correlaciones entre estas variables, sus dimensiones e indicadores utilizando el coeficiente de Pearson.

\section{Resultados de la investigación}

En la Tabla 1 se presentan los resultados globales para el liderazgo desde la perspectiva del líder. Para la dimensión transformador el promedio de 2.66 que se interpreta como media alta, la desviación fue de 0.53 y la varianza de 0.29 , que indica una baja dispersión de los puntajes. El mínimo fue de 0.76 y el máximo de 3.90. En cuanto al liderazgo transaccional, el promedio fue de 1.72 que se interpreta como media baja, la desviación fue de 0.57 y la varianza de 0.33 , que indica una baja dispersión de los puntajes. El mínimo fue de 0.38 y el máximo de 3.80. Y en relación a los resultados del liderazgo, el promedio es de 2.71 que se interpreta como media alta, la desviación fue de 0.58 y la varianza de 0.34 , que indica una baja dispersión de los puntajes. El mínimo fue de 0.67 y el máximo de 4.00 .

Por otra parte en cuanto al liderazgo desde la perspectiva del seguidor (ver Tabla 2), para la dimensión transformador se encontró un promedio de 2.26 que se interpreta como media alta, la desviación fue de 0.81 y la varianza de 0.66 , que indica una media dispersión de los puntajes. El mínimo fue de 0.00 y el máximo de 4.00. Para el liderazgo transaccional, el

Tabla 1

Estadísticas descriptivas para la variable liderazgo-perspectiva del líder

\begin{tabular}{lcccccc}
\hline Dimensión & Promedio & $\begin{array}{c}\text { Desviación } \\
\text { Estándar }\end{array}$ & Varianza & Mínimo & Máximo & Categoría \\
\hline Transformador & 2,66 & 0,53 & 0,29 & 0,76 & 3,90 & Media Alta \\
Transaccional & 1,72 & 0,57 & 0,33 & 0,38 & 3,80 & $\begin{array}{c}\text { Media } \\
\text { Baja }\end{array}$ \\
Resultados & 2,71 & 0,58 & 0,34 & 0,67 & 4,00 & Media Alta \\
\hline
\end{tabular}


Liderazgo y cultura organizacional en instituciones de Educación Básica Pirela de Faría, Ligia del Carmen

promedio fue de 1.78 que se interpreta como media baja, la desviación fue de 0.66 y la varianza de 0.44 , que indica una baja dispersión de los puntajes. El mínimo fue de 0.00 y el máximo de 3.40 . Y para la dimensión resultados del liderazgo el promedio de 2.31 que se interpreta como media alta, la desviación fue de 0.98 y la varianza de 0.96 , que indica una media dispersión de los puntajes. El mínimo fue de 0.00 y el máximo de 4.00 .

Por lo tanto, existe una mayor tendencia a percibir un liderazgo transformador tanto en si mismos como en los líderes, así como en los resultados de satisfacción, efectividad y esfuerzo extra, que un liderazgo transaccional. De manera que consideran que el líder proporciona visión y sentido de misión, origina orgullo, genera respeto y confianza, otorga a sus colaboradores un sentido de propósito, constituye un modelo que los colaboradores aspiran a imitar. Asimismo comunica altas expectativas, expresando los propósitos importantes de manera sencilla, promueve y enfatiza la inteligencia, racionalidad y solución cuidadosa de problemas, estimulando a sus colaboradores a reconsiderar sus propias ideas. Dan atención personal, trata a cada empleado de manera individual, capacitando, aconsejándolo.
Se entiende entonces el liderazgo transformador aquel que reconoce y explota una necesidad o demanda personal existente de un seguidor potencial, buscando motivos potenciales, satisfaciendo altas necesidades, y envolviendo a la persona completa del seguidor, toman responsabilidad por sus compromisos, es decir, cumplen lo que prometen. El resultado del liderazgo es una relación de estimulación mutua que convierte seguidores en líderes y puede convertir líderes en agentes morales. Los líderes y seguidores tienen una relación no sólo de poder sino de necesidades, aspiraciones y valores mutuos (Burns, 2003).

A continuación se presentan los resultados para la variable cultura organizacional (ver Tabla 3), entendida como un patrón de supuestos básicos inventados, descubiertos y/o desarrollados por un grupo, en la medida que aprende a manejar y resolver sus problemas de adaptación externos y de integración interna. Para el indicador formalidad, el promedio fue de 3.24 que se interpreta como media alta, la desviación fue de 0.64 y varianza de 0.40 , indicando una dispersión media de los puntajes. Asimismo para el indicador estilo de dirección, el promedio fue de 3.00 que se interpreta como media alta, la desviación

Tabla 2

Estadísticas descriptivas para la variable liderazgo-perspectiva del seguidor

\begin{tabular}{lcccccc}
\hline Dimensión & Promedio & $\begin{array}{c}\text { Desviación } \\
\text { Estándar }\end{array}$ & Varianza & Mínimo & Máximo & Categoría \\
\hline Transformador & 2,26 & 0,81 & 0,66 & 0,00 & 4,00 & Media Alta \\
Transaccional & 1,78 & 0,66 & 0,44 & 0,00 & 3,40 & Media Baja \\
Resultados & 2,31 & 0,98 & 0,96 & 0,00 & 4,00 & Media Alta \\
\hline
\end{tabular}


fue de 0.76 y varianza de 0.57 , indicando una dispersión media de los puntajes. En cuanto al indicador integración organizacional, el promedio fue de 3.04, ubicada en el nivel medio alto. La desviación fue de 0.66 y varianza de 0.43 , indicando una dispersión media de los puntajes.

En relación al indicador desarrollo de recursos humanos, el promedio fue de 3.18, ubicado en la categoría media alta, la desviación fue de 0.64 y varianza de 0.41 , indicando una dispersión media de los puntajes. En el indicador recompensas y beneficios, el promedio fue de 2.55 , ubicado en la categoría media alta, la desviación fue de 0.71 y varianza de 0.51 , indicando una dispersión media de los puntajes. Para el indicador sistemas de apoyo, el promedio fue de 2.79 , ubicado en la categoría media alta. La desviación fue de 0.78 y varianza de 0.61 , indicando una dispersión media de los puntajes.

En cuanto al indicador compromiso, el promedio fue de 3.28 , ubicado en la categoría alta. La desviación fue de 0.56 y varianza de 0.32 , indicando una disper- sión media de los puntajes. Para la orientación a resultados, el promedio fue de 2.94, ubicado en la categoría media alta. La desviación fue de 0.62 y varianza de 0.38 , indicando una dispersión media de los puntajes.

Estos resultados permiten visualizar que tanto los docentes como los directores perciben en un nivel medio alto, claridad de la estructura, objetivos, metas, procedimientos y responsabilidades. El estilo de dirección da estímulo, confianza, participación en la toma de decisiones y comunicación. Existe comunicación lateral y cooperación intra e intergrupos. Asimismo se perciben condiciones de trabajo, recursos y tecnología para llevar a cabo el trabajo y un alto sentido de pertenencia a la organización y satisfacción con el trabajo. Sin embargo, se perciben en menor grado el desarrollo del recurso humano en cuanto a los sistemas de evaluación, adiestramiento, oportunidades de crecimiento, la compensación y recompensas, y la preocupación por alcanzar los objetivos y metas.

Tabla 3

Estadísticas descriptivas para la variable cultura organizacional

\begin{tabular}{lcccccc}
\hline Indicador & $\begin{array}{c}\text { Promedio } \\
\text { Desviación }\end{array}$ & $\begin{array}{c}\text { Varianza } \\
\text { Estándar }\end{array}$ & Mínimo & Máximo & Categoría \\
\hline Formalidad & 3,24 & 0,64 & 0,40 & 1,00 & 4,00 & Medio Alto \\
Estilo de Dirección & 3,00 & 0,76 & 0,57 & 1,00 & 4,00 & Medio Alto \\
Integración Organizacional & 3,04 & 0,66 & 0,43 & 1,00 & 4,00 & Medio Alto \\
$\begin{array}{l}\text { Desarrollo de recursos } \\
\text { humanos }\end{array}$ & 3,18 & 0,64 & 0,41 & 1,00 & 4,00 & Medio Alto \\
Recompensas y beneficios & 2,55 & 0,71 & 0,51 & 1,00 & 4,00 & Medio Alto \\
Sistemas de apoyo & 2,79 & 0,78 & 0,61 & 1,00 & 4,00 & Medio Alto \\
Compromiso & 3,28 & 0,56 & 0,32 & 1,40 & 4,00 & Medio Alto \\
Orientación a resultados & 2,94 & 0,62 & 0,38 & 1.00 & 4,00 & Medio Alto \\
\hline
\end{tabular}


Liderazgo y cultura organizacional en instituciones de Educación Básica Pirela de Faría, Ligia del Carmen

En cuanto a las correlaciones entre las dimensiones del liderazgo (perspectiva del líder) y la cultura organizacional, estas fueron bajas, menores de 0.24, siendo significativas para la dimensión transformador con los indicadores formalidad, integración organizacional, recompensa y beneficios, y orientación a resultados. La dimensión resultados del liderazgo correlacionó significativamente aunque bajo con formalidad y orientación a resultados. Mientras que el liderazgo transaccional correlacionó bajo aunque significativo y negativo con Sistemas de apoyo. Es decir que a mayor percepción de un liderazgo transformador mayor será la formalidad, la comunicación, las recompensas y beneficios y la orientación a resultados. Mientras mas se percibe un liderazgo transaccional menor son los sistemas de apoyo, y a medida que los resultados del liderazgo sean mas positivos, mayor será la formalidad y la orientación a los resultados de la cultura organizacional (ver Tabla 4).

En cuanto a las correlaciones entre las dimensiones del liderazgo transfor- mador, desde la perspectiva del seguidor y los indicadores de la cultura organizacional, estas fueron medias y significativas. Tanto el liderazgo transformador como los resultados correlacionaron significativamente aunque media con todos los indicadores de la cultura. Mientras que el liderazgo transaccional no correlacionó con ninguno de los indicadores de la cultura (ver Tabla 5). Por lo tanto en líneas generales la percepción de un liderazgo transformador como los resultados del liderazgo están más asociados a una percepción más positiva de la cultura de la organización, no así con el liderazgo transaccional.

\section{Conclusiones}

En relación a los resultados evidenciados en los docentes y directores, con respecto al liderazgo, el estilo transformador desde la perspectiva del líder y como seguidor, se encontró que esta resultó ser media alta con una media dispersión, de manera que perciben al lídery a si mismos con influencia idealizada

Tabla 4

Correlaciones entre el liderazgo (perspectiva del líder) y la cultura organizacional

\begin{tabular}{|c|c|c|c|c|c|c|c|c|c|}
\hline Indicadores & & Formal. & $\begin{array}{l}\text { Estilo } \\
\text { direc. }\end{array}$ & $\begin{array}{l}\text { Integr. } \\
\text { Organiz. }\end{array}$ & $\begin{array}{c}\text { Desarr. } \\
\text { Rec. } \\
\text { Hum }\end{array}$ & $\begin{array}{c}\text { Recomp. } \\
\text { Benef. }\end{array}$ & $\begin{array}{l}\text { Sist. } \\
\text { apoyo }\end{array}$ & Comprom. & $\begin{array}{l}\text { Orient. } \\
\text { Result. }\end{array}$ \\
\hline \multirow[t]{2}{*}{ Transformador } & $\mathrm{C}$ & 0,17 & 0,05 & 0,13 & 0,05 & 0,14 & 0,08 & 0,05 & 0,24 \\
\hline & $\mathrm{P}$ & 0,00 & 0,38 & 0,02 & 0,40 & 0,02 & 0,18 & 0,41 & 0,00 \\
\hline \multirow[t]{2}{*}{ Transaccional } & C & $-0,01$ & $-0,02$ & $-0,06$ & $-0,03$ & 0,07 & $-0,12$ & $-0,09$ & 0,01 \\
\hline & $\mathrm{P}$ & 0,93 & 0,74 & 0,30 & 0,62 & 0,27 & 0,04 & 0,12 & 0,90 \\
\hline \multirow[t]{2}{*}{ Resultados } & C & 0,15 & 0,03 & 0,08 & 0,11 & 0,04 & 0,05 & 0,02 & 0,16 \\
\hline & $P$ & 0,02 & 0,65 & 0,18 & 0,07 & 0,49 & 0,42 & 0,70 & 0,01 \\
\hline
\end{tabular}

C: correlación. P: probabilidad. 
Tabla 5 Correlaciones entre el liderazgo (seguidor) y la cultura organizacional

\begin{tabular}{|c|c|c|c|c|c|c|c|c|c|}
\hline Indicadores & & Formal. & $\begin{array}{l}\text { Estilo } \\
\text { direc. }\end{array}$ & $\begin{array}{l}\text { Integr. } \\
\text { Organiz. }\end{array}$ & $\begin{array}{c}\text { Desarr. } \\
\text { Rec. } \\
\text { Hum }\end{array}$ & $\begin{array}{l}\text { Recomp. } \\
\text { Benef. }\end{array}$ & $\begin{array}{l}\text { Sist. } \\
\text { apoyo }\end{array}$ & Comprom. & $\begin{array}{l}\text { Orient. } \\
\text { Result. }\end{array}$ \\
\hline \multirow[t]{2}{*}{ Transformador } & $\mathrm{C}$ & 0,25 & 0,37 & 0,28 & 0,35 & 0,26 & 0,28 & 0,24 & 0,32 \\
\hline & $\mathrm{P}$ & 0,00 & 0,00 & 0,00 & 0,00 & 0,00 & 0,00 & 0,00 & 0,00 \\
\hline \multirow[t]{2}{*}{ Transaccional } & $\mathrm{C}$ & 0,03 & 0,03 & 0,05 & 0,06 & 0,08 & 0,02 & 0,05 & 0,00 \\
\hline & $\mathrm{P}$ & 0,60 & 0,65 & 0,44 & 0,29 & 0,18 & 0,75 & 0,43 & 0,97 \\
\hline \multirow[t]{2}{*}{ Resultados } & $\mathrm{C}$ & 0,30 & 0,42 & 0,30 & 0,45 & 0,28 & 0,28 & 0,32 & 0,31 \\
\hline & $\mathrm{P}$ & 0,00 & 0,00 & 0,00 & 0,00 & 0,00 & 0,00 & 0,00 & 0,00 \\
\hline
\end{tabular}

C: correlación. P: probabilidad.

como atributo, y comportamiento, estimulación intelectual, consideración individualizada y motivación inspiradora. El estilo transaccional autopercibido y percibido en el supervisor, fueron medio bajo, caracterizado por recompensas contingentes, gerencia por excepción activa y pasiva, y laissez faire. En cuanto a la dimensión resultados, fue media alta con una baja dispersión de los puntajes tanto para la autopercepción como seguidor que se enfocan en esfuerzo extra, efectividad y satisfacción los resultados.

En este sentido, se plantea que el liderazgo reconoce y explota una necesidad o demanda personal existente de un seguidor potencial, buscando motivos potenciales, satisfaciendo altas necesidades, y envolviendo a la persona completa del seguidor. Los líderes toman responsabilidad por sus compromisos, es decir, cumplen lo que prometen. El resultado del liderazgo es una relación de estimulación mutua que convierte seguidores en líderes y puede convertir líderes en agentes morales. Los líderes y seguidores tienen una relación no sólo de poder sino de necesidades, aspiraciones y valores mutuos. En respuesta a los líderes, los seguidores tienen un adecuado conocimiento de los programas alternativos propuestos por el líder y la capacidad para escoger entre ellos.

Es decir que los líderes son mas transformadores, primariamente en términos de los efectos del líder sobre sus seguidores, quienes sienten confianza, admiración, fidelidad, respeto hacia el líder y son motivados a hacer mas de los que se espera de ellos.

Por otro parte, en referencia a la cultura organizacional, el indicador formalidad, resultó ser medio alto con una dispersión media, al Igual que para los indicadores estilo de dirección, integración organizacional, desarrollo de recursos humanos, sistemas de apoyo, compromiso, recompensas y beneficios, y orientación a resultados. En concordancia con esto la cultura se entiendo como el conjunto (sistema) de valores, tradiciones, creencias, hábitos, normas, actitudes y conductas que dan identidad, personalidad y destino a una organización para el 
Liderazgo y cultura organizacional en instituciones de Educación Básica Pirela de Faría, Ligia del Carmen

logro de sus objetivos sociales como lo es la formación de las personas.

De manera que los aspectos relacionados con la cultura como la formalidad, está referida a la claridad de la estructura, objetivos, metas, procedimientos y responsabilidades, así como el estilo de dirección, relacionado con el estímulo, confianza, participación en la toma de decisiones y comunicación. La integración organizacional, o la comunicación lateral, cooperación intra e intergrupos, el desarrollo del recurso humano, basado en la evaluación, adiestramiento y las oportunidades de crecimiento.

La compensación y recompensas están asociadas a la equidad interna y externa, beneficios, estabilidad laboral, relación de las recompensas con el desempeño. Los sistemas de apoyo, se refieren a las condiciones de trabajo, recursos y tecnología para llevar a cabo el trabajo. El compromiso al sentido de pertenencia a la organización y satisfacción con el trabajo. Y la orientación a resultados, a la preocupación por alcanzar los objetivos y metas.

Se encontraron las correlaciones bajas y algunas significativas desde el punto de vista del líder, entre las dimensiones del liderazgo, específicamente el transformador (líder) y los resultados con la cultura organizacional. Mientras que desde el punto de vista del seguidor, estas fueron medias, aunque la mayoría significativas. No se encontraron correlaciones entre el estilo transaccional con la cultura organizacional.

Lo que quiere decir que a medida que la percepción del líder sea mas transformadora, que reconozca y satisfaga altas necesidades de los seguidores, to- mando responsabilidad por los compromisos asumidos, generando confianza, admiración, fidelidad, respeto hacia el líder y motivación a superar las expectativas, mejor será la percepción que tienen de la cultura en referencia a la formalidad, el estilo de dirección, comunicación, el desarrollo de recursos humanos, los sistemas de apoyo, el compromiso, recompensas y beneficios, y orientación a resultados.

\section{Referencias Bibliográficas}

Bass Bernard y Avolio Bruce (1994). Improving Organizational Effectiveness throght transformational Leadership. Sage Publications, Inc. USA.

Bass, Bernard y Avolio, Bruce (2004). Multifactor Leadership Questionnaire. En www.mindgarden.com/products/mlq. html [Fecha de Consulta: 27/07/ 2007].

Bennis, Warren y Nanus, Burt (1985). Lideres. Las cuatro claves del liderazgo eficaz. Edit. Norma.

Bower, Joseph (1995). Oficio y Arte de la Gerencia. Volumen I. Escuela de Administración de Empresas Harvard. Grupo Editorial Norma. Colombia.

Burns, James (2003). Transforming Leadership. Grove Press, New York.

Daft, Richard (2006). La experiencia del liderazgo. Edit. Thomson. 3ra. Edición. México

Dilts, Robert (1998). Liderazgo Creativo. Edit. Urano. Barcelona.

García, Salvador y Dolan, Shimon (1997). La dirección por Valores. Edit. McGraw Hill. Madrid, España.

Gordon, Judith (1997). Comportamiento Organizacional. Edit. Prentice Hall. México. 
Granel, Elena; Garaway, David y Malpica, Claudia (1998). Éxito gerencial y cultura: Retos y oportunidades en Venezuela. Ediciones IESA, Venezuela.

Hernández, Roberto; Fernández, Carlos y Baptista, Pilar (2000). Metodología de la Investigación. Edit. McGraw Hill. México.

Hellrriegel, D. y Slocum, J. (1998). Comportamiento Organizacional. Edit. Thomson learning. México.

Lussier, Robert y Achua, Christopher (2002). Liderazgo. Teoría-aplicación-Desarrollo de Habilidades. Edit. Thomson Learning, México.

Robbins, Stephen (2004). Comportamiento Organizacional. Teoría y Práctica. Edit. Prentice Hall. México.
Serna, Humberto (1997). Gerencia Estratégica: Planeación y Gestión - Teoría y Metodología. 3R Editores. Bogotá.

Schein, Edgar (1992). Cultura Organizacional y Liderazgo. Edit Plaza y Janes. Barcelona.

Sierra, Restituto (1999). Técnicas de Investigación Social. Edit. Paraninfo, México.

Siliceo, Alfonso; Casares, David y González, José (1999). Liderazgo, Valores y Cultura organizacional. Hacia una Organización Competitiva. Edit. Mc Graw Hill, México.

Stoner, James (1996). Administración. Edit. Prentice Hall, México. 